\title{
Motion Segmentation using Mathematical Morphology
}

\author{
Arnaldo Câmara Lara and Roberto Hirata Jr. \\ University of São Paulo \\ Instituto de Matemática e Estatística \\ Rua do Matão, 1010, 05508-090, São Paulo, Brazil \\ (alara,hirata)@ime.usp.br
}

\begin{abstract}
Motion segmentation is one of the first steps in image sequence applications. The problem has been extensively studied and approached in several ways but it remains a difficult task even for sequences acquired by stationary cameras. In this paper we propose a novel algorithm that is based on Mathematical Morphology. The algorithm is simple to be implemented, less subject to lighting changes and more robust to false positives. It has been tested in security cameras' image sequences showing promising results. Some algorithms from literature have been implemented and compared to the one described here.
\end{abstract}

\section{Introduction}

Motion segmentation is vital to several video applications because it will delimit regions of interest to the analysis phases. The problem has been approached extensively in the last two decades but it remains a difficult task [23] even when videos are acquired by stationary cameras.

There are basically four approaches to treat this problem [24]: 1) background modelling and subtraction, 2) statistical point analysis, 3) temporal differentiation and 4) optical flow.

1. In videos generated by stationary cameras, motion segmentation can be achieved by analyzing each new frame in relation to a model of the scene background. Usually, the symmetric difference operator [3] is used because it detects pixels that changed in the current frame in relation to the background model. For instance, the pixels of a chair that was in the model and is right shifted in the current frame. Some advantages of this approach are its simplicity and the good shape recovery of the moving objects. Some problems are the susceptibility to lighting changes and the computational cost to create and keep the background model [23]. A trivial solution to estimate the background is to film the scenery without moving objects or to set a specific color to the background as in the Chroma keying technique [7]. Both solutions are impossible in uncontrolled scenes. A more realistic solution is using a subset of the initial frames of the image sequence to estimate the background using some statistics like mean or median. The mean is a simple and efficient statistics, but the most susceptible to discrepant values. The median is a more robust statistics [24], however, median implies in more computational costs and memory usage. Adaptive mean has already been tried [4] to reestimate the background at each frame, and it is more robust to lighting changes.

2. In the statistical point analysis approach, statistics are computed and maintained for each pixel based on an interval of time of the video. The pixels are classified as background or foreground according to arbitrary thresholds or statistical hypothesis tests. One example of this technique is the use of the Wiener filter in Wallflower algorithm [23]. Techniques that use this approach are very robust and popular nowadays.

3. In the temporal differentiation approach, the most used technique is to compute the difference between two consecutive frames. It is simple and robust to lighting changes, but it recovers a very poor shape of the foreground and it is very susceptible to noise. One way to reduce noise is to use the information of three consecutive frames, as in [4]. The complete shape of the target objects can be obtained by using a connected operator [5].

4. Optical Flow is defined as a 2D distribution of apparent displacement vectors among pixels of consecutive frames [21] and it is used to describe a coherent movement of points or features in an image sequence. The optical-flow-based segmentation uses features of the vectors to find regions of similar displacement. A 
problem in this approach is the computational cost that inhibits its use in a real-time application without specialized hardware.

Combinations of the four approaches above have been tried, for instance, in [4], the authors use a hybrid approach mixing temporal differentiation and background subtraction obtaining good results; in [1], the authors use temporal differentiation between the gradient [9] of two consecutive frames and a 3D human model is fitted into the result (to obtain a real-time performance, specialized hardware has been designed and used).

In this paper, the problem of motion segmentation is treated by a novel algorithm based on the background modelling and subtraction approach. The algorithm uses mainly the geometrical information of the scene extracted by operators defined by Mathematical Morphology (MM). The algorithm has been tested on two different public video sets of the CAVIAR project [10] in more than 38 video samples showing good preliminaries results. The proposed approach is simple (it uses very simple MM operations and operators), fast, robust to noise and most important, the results are less susceptible to lighting changes.

After this Introduction, we review some basic Mathematical Morphology operations and operators used in this work in Section 2. In Section 3, we present the proposed algorithm. In Section 4, we present the experimental results and in Section 5, we present some conclusions of this work.

\section{Mathematical Morphology}

Mathematical Morphology (MM) is a branch of nonlinear image processing and analysis [5] developed initially by Matheron [15] and Serra [20]. It is also a solid algebraic theory to study transformations between complete lattices [2, 3]. In this section, we review the most important MM concepts used in the proposed algorithm.

Let $Z$ be the set of integers, $k \in Z, k>0, E$ be a subset of $Z^{2}$ and $K$ be the interval $[0, k-1] \subset Z$. A gray-scale image $f$ can be represented as an application from $E$ to $K$, $f: E \longrightarrow K$. The set of all images from $E$ to $K$ is also denoted by $K^{E}$. An image sequence $\mathcal{V}$ is an ordered list of images, $\left(f_{1}, f_{2}, \ldots, f_{n}\right)$, where each item $f_{i} \in K^{E}$ is the $i^{t h}$ frame in the image sequence.

Let $B$ be a subset of $E$ and $b$ a function from $B$ to $Z$. We will call $B$ a structuring element and $b$ a structuring function. When the structuring function is defined from $B$ to $\{-\infty, 0\}$ it is called flat structuring element (in contrast to non-flat structuring element) and it is denoted simply by $B$. Structuring elements and functions play an important role in the definition of dilations and erosions, two MM elementary operators.

To define dilation and erosion by a structuring function $b$, one needs to define the reflection, the dot sum and the dot subtraction [3]. The reflection of $b$, denoted $\breve{b}$, is given by $\breve{b}(x)=b(-x)$. Let $\dot{+}$ be an operation defined in $K \times Z$ to $Z$, by, any $t \in K$ and $v \in Z$ :

$$
t \dot{+} v=\left\{\begin{array}{cl}
0 & \text { if } t=0 \\
0 & \text { if } t>0 \text { and } t+v \leq 0 \\
t+v & \text { if } t>0 \text { and } 0 \leq t+v \leq k \\
k & \text { if } t>0 \text { and } t+v>k
\end{array}\right.
$$

Similarly, we can define the $\dot{-}$ operation by:

$$
t \dot{-} v=\left\{\begin{array}{cl}
0 & \text { if } t<k \text { and } t-v \leq 0 \\
t-v & \text { if } t<k \text { and } 0 \leq t-v \leq k, \\
k & \text { if } t<k \text { and } t-v>k \\
k & \text { if } t=k .
\end{array}\right.
$$

The dilation of a gray-scale image $f$ by the structuring function $b$ is the function $f \oplus b$ defined for any $x \in E$ as:

$$
(f \oplus b)(x)=\max \{f(y) \dot{+} b(x-y): y \in(\breve{B}+x) \cap(E)\}
$$

Similarly, the erosion of a function $\mathrm{f}$ by the structuring function $\mathrm{b}$ is the function $f \ominus b$ defined for any $x \in E$ :

$$
(f \ominus b)(x)=\min \{f(y) \dot{-} b(x-y): y \in(B+x) \cap(E)\}
$$

Erosions and dilations can be combined in different manners producing new operators [3]. Closings and openings are the result of a trivial composition of a dilation and an erosion by the same structuring function:

$$
\begin{aligned}
f \circ b & =(f \ominus b) \oplus b \text { (opening) } \\
f \bullet b & =(f \oplus b) \ominus b \text { (closing) }
\end{aligned}
$$

Conditional erosion of a function $f$ by a structuring element $b_{c}$ subject to a marker $g \in K^{E}$ is the function $f \ominus_{g} b_{c}$ defined as:

$$
f \ominus_{g} b_{c}=\left(f \ominus b_{c}\right) \vee g,
$$

where $\vee$ is the union operation. Conditional dilation of $f$ by the structuring element $b_{c}$ conditioned to the marker $g$ is the function $f \oplus_{g} b_{c}$ defined as:

$$
f \oplus_{g} b_{c}=\left(f \oplus b_{c}\right) \wedge g
$$

where $\wedge$ is the intersection operation. The structuring element $b_{c}$ defines the connectivity to be used in the operation: a $3 \times 3$ cross defines a 4 -connectivity and a $3 \times 3$ box defines a 8-connectivity [5]. A sequence of $n$ conditional erosions and dilations are defined respectively as:

$$
\begin{aligned}
& \left(f \ominus_{g} b_{c}\right)^{n}=\left(\left(\left(f \ominus_{g} b_{c}\right) \ominus_{g} b_{c}\right) \ominus_{g} \ldots \ominus_{g} b_{c}\right),(n \text { times }) \\
& \left(f \oplus_{g} b_{c}\right)^{n}=\left(\left(\left(f \oplus_{g} b_{c}\right) \oplus_{g} b_{c}\right) \oplus_{g} \ldots \oplus_{g} b_{c}\right),(n \text { times })
\end{aligned}
$$

The sup-reconstruction $f \nabla_{b_{c}} g$ and the inf-reconstruction $f \triangle_{b_{c}} g$ operators of a image $f$ by a marker $g$ using connectivity defined by $b_{c}$ are conditional erosions and dilations 
applied until the stability or the result of the $i^{t h}$ application is equal the previous result.

$$
\begin{gathered}
f \nabla_{b_{c}} g=\left(g \ominus_{f} b_{c}\right)^{\infty} \text { (sup-reconstruction) } \\
f \triangle_{b_{c}} g=\left(g \oplus_{f} b_{c}\right)^{\infty} \text { (inf-reconstruction) }
\end{gathered}
$$

The closing and opening by reconstruction are defined utilizing sup- and inf-reconstructions in their implementation.

$$
\begin{gathered}
f \bullet_{b_{c}} g=(f \bullet g) \nabla_{b_{c}} f \text { (closing by reconstruction) } \\
f \circ_{b_{c}} g=(f \circ g) \triangle_{b_{c}} f \text { (opening by reconstruction) }
\end{gathered}
$$

The $h$-Min and $h$-Max operators eliminates basins and peaks with contrast less then a specified parameter $h$. A structuring element $b_{c}$ is used to specify the connectivity to be used. These operators are also implemented using the previous sup- and inf-reconstructions operators.

$$
\begin{aligned}
h-\operatorname{Min}_{h, b_{c}}(f) & =(f+h) \nabla_{b_{c}} f \\
h-\operatorname{Max}_{h, b_{c}}(f) & =(f-h) \triangle_{b_{c}} f
\end{aligned}
$$

Analogously, the $v$-Min and $v$-Max operators eliminates basins and peaks with volumes less then a specified parameter $v$. They are also implemented using the previous supand inf-reconstructions operators.

The edges of an object can be detected by morphological operators. External gradient $\operatorname{grad}_{g_{e}}^{e x t}$ produces a external edges and is defined as:

$$
\operatorname{grad}_{g_{e}}^{e x t}(f)=\left(f \oplus g_{e}\right)-f
$$

The internal gradient produces internal edges:

$$
\operatorname{grad}_{g_{i}}^{i n t}(f)=f-\left(f \ominus g_{i}\right)
$$

And the morphological gradient is:

$$
\operatorname{grad}_{g_{e}, g_{i}}(f)=\left(f \oplus g_{e}\right)-\left(f \ominus g_{i}\right)
$$

The opening top-hat operator extracts the peaks of an image. It is defined by the morphological subtraction between the image $f$ and the opening of same $f$ by the structuring element $\mathrm{b}$,

$$
f \hat{o} b=f-(f \circ b) .
$$

The opening by reconstruction top-hat operator also extracts peaks of an image, but keeping the original contours. It is defined by the morphological subtraction between the image $f$ and the opening by reconstruction of the same image $f$ by the structuring element $b_{c}$,

$$
f \hat{\circ}_{b_{c}} b=f-\left(f \circ_{b_{c}} b\right)
$$

where $b_{c}$ is a structuring element that specifies the used connectivity.
A connected operator is an operator that preserves the edges in the result image, i.e., all the edges in the result image were in the original image. It does not create new edges in the original image. A flat-zone in an image is a connected region with a constant gray-level. A connected operator always decrease the number of flat-zone in an image. This is useful to the segmentation process.

\section{The Proposed Algorithm}

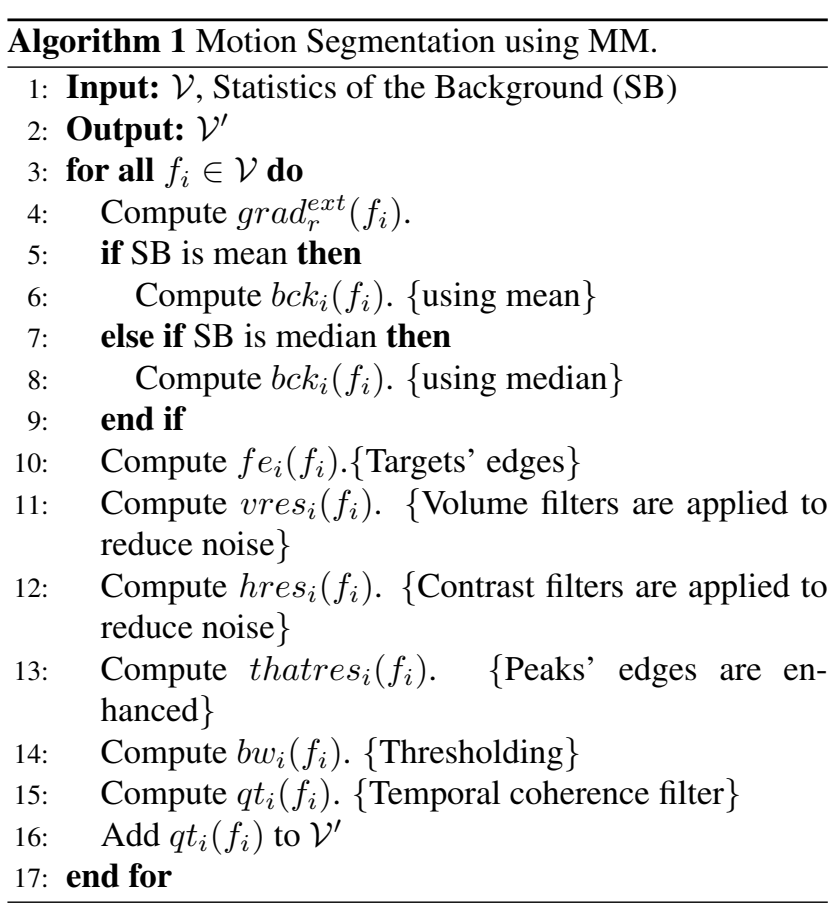

The proposed algorithm is based on the background modeling and subtraction approach. Algorithm 1 shows a high-level description of the proposed algorithm. It receives as input a gray-scale image sequence $\mathcal{V}$ and saves an output image sequence $\mathcal{V}^{\prime}$ containing the foreground objects of the input image sequence. The idea and its implementation are straightforward and can be followed in the diagram of the solution (Fig. 1). For each frame $f_{i}$ of $\mathcal{V}$, the morphological gradient of the current frame is computed, the background model is estimated and a subtraction is performed to get an approximation of the foreground. After that, the resulting image is filtered using morphological filters, mainly connected operators are applied to reduce noise. Connected operators do not create new edges in the resulting image, i.e, all the edges in the resulting images are in the original image. Therefore, they are important to preserve the most significant edges while filtering the irrelevant ones. Peaks are enhanced, and a binary image of the last result is produced and, finally, regions that do not show a temporal coherence are eliminated. The result reveals an approximation 


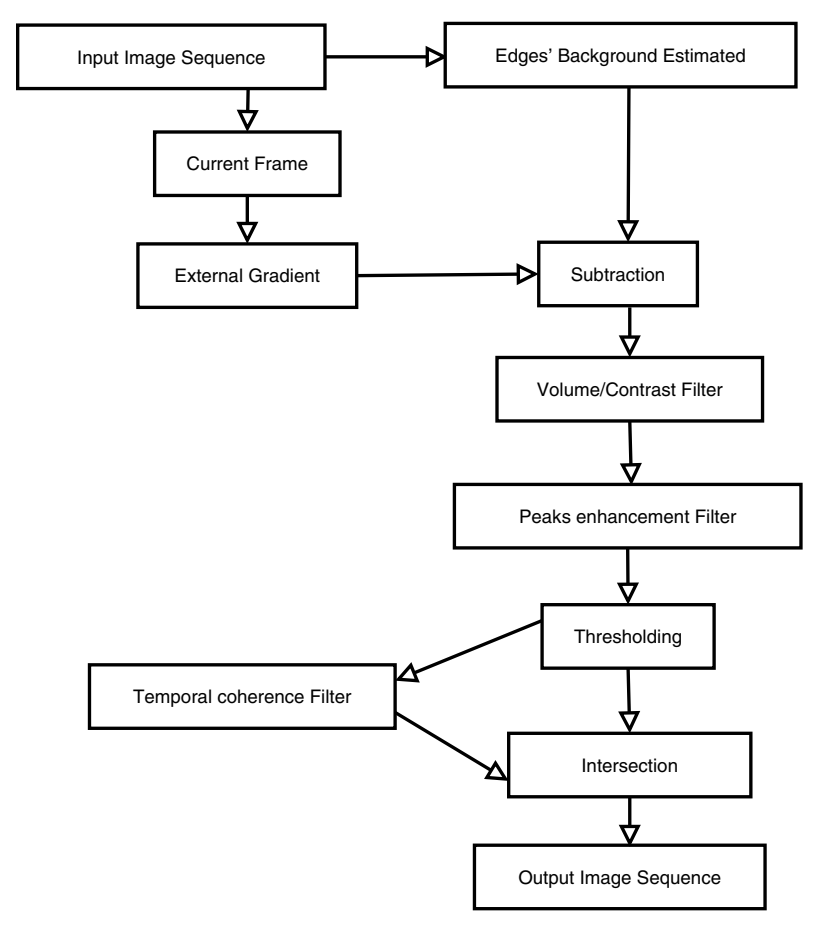

Figure 1. Diagram of the solution.

of the foreground's edges.

Let $f_{i} \in \mathcal{V}$ be an image of the sequence and let $r$ be the non-flat structuring element represented in Table 1. From a topographical perspective, dilating or eroding an image by $r$ will modify it in all the directions uniformly. So, the external gradient using $r$ produces a better model of the moving borders than when using a flat-structuring element. Denoting by bck ${ }_{i}$ the background model based on the morphological gradient, we compute the mean, or the median, of all frames up to frame $i$ (a variation that is being tested computes the mean or the median from frame $i_{0}$ to $i$, where $1 \leq i_{0}<i$ ). Formally, let $I$ be the interval $[1, i]$ and $|I|$, the number of frames in the interval (in this case i),

$$
\begin{gathered}
\operatorname{bck}_{i}^{m}=\frac{1}{|I|} \sum_{j=1}^{i} \operatorname{grad}_{r}^{e x t}\left(f_{i}\right) \\
\operatorname{bck}_{i}^{M}=\operatorname{Median}\left(\operatorname{grad}_{r}^{e x t}\left(f_{I}\right)\right),
\end{gathered}
$$

where $\operatorname{grad}_{r}^{\text {ext }}\left(f_{I}\right)$ means we consider all the gradient results of the sequence up to frame i. Notice that we have introduced a letter $m$ or a letter $M$ to the notation to distinguish mean and median, respectively. The median computation is optimized by using a special data structure that holds the histogram of each pixel.

To have an estimate of the moving objects, we compute the morphological subtraction of the background model from the gradient of the current frame denoted by $f e_{i}$.

\begin{tabular}{|l|l|l|}
\hline 1 & 2 & 1 \\
\hline 2 & 3 & 2 \\
\hline 1 & 2 & 1 \\
\hline
\end{tabular}

Table 1. Non-flat structuring element.

$$
f e_{i}=\operatorname{grad}_{r}^{e x t}\left(f_{i}\right)-b c k_{i}^{s}
$$

where $s$ can be the letter $m$ or $M$.

To reduce noise, we first compose the morphological filters $v$-Min and $v$-Max to eliminate, respectively, basins and peaks with a volume less than a specified argument $V_{\min }$.

$$
\text { vres }_{i}=v-\operatorname{Max}_{V_{\text {min }}, b_{c}}\left(v-\operatorname{Min}_{V_{\text {min }}, b_{c}}\left(f e_{i}\right)\right)
$$

where the structuring element $b_{c}$ is a cross $(3 \times 3)$ meaning that the operator uses a 4-connectivity.

Next, we compose the morphological filters $h$-Min and $h$-Max to eliminate, respectively, basins and peaks with contrast less than a specified parameter $H_{\text {min }}$. The same cross $b_{c}$ is used as the structuring element.

$$
h r e s_{i}=h-\operatorname{Max}_{H_{m i n}, b_{c}}\left(h-\operatorname{Min}_{H_{m i n}, b_{c}}\left(\text { vres }_{i}\right)\right)
$$

To enhanced the regions of the moving edges of the image, an opening by reconstruction top-hat operator is used to obtain the peaks of $h r e s_{i}$. The structuring element used, $d$, is a flat disk with radius 3 and the same structuring element $b_{c}$.

$$
\text { thatres }_{i}=\text { hres }_{i} \hat{\mathrm{o}}_{c} d
$$

The result is finally converted to binary using a trivial threshold 1.

$$
b w_{i}=\text { Th }\left(\text { thatres }_{i}, 1\right)
$$

where $T h$ represents the threshold operator [9].

For very low-quality videos, some edges that do not belong to the moving objects will be in $b w_{i}$, for some $i$. A possible solution to this problem is to use a heuristics based on time coherence that helps to keep just the edges that were approximately in the same position for some previous frames. Because of the small displacement of the moving objects between a frame and the previous, $b w_{i-1}$ and $b w_{i-2}$ are dilated by a flat disk with radius 3 and used as a previous information to keep the edges of $b w_{i}$. The following formula formalizes this coherence operator,

$$
q t_{i}=b w_{i} \wedge\left(b w_{i-1} \oplus c\right) \wedge\left(b w_{i-2} \oplus c\right),
$$

where $\wedge$ is the intersection operation [5]. The final result $q t_{i}$ is added to the video output $\mathcal{V}^{\prime}$. 


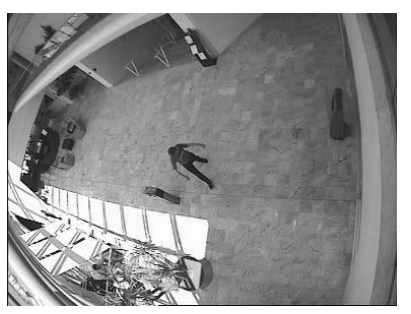

(a) Original Frame

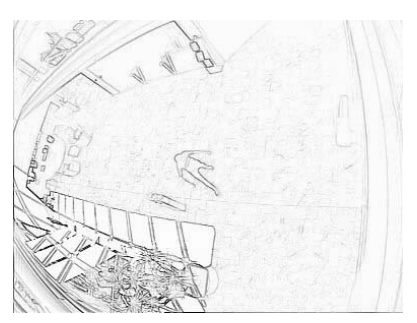

(c) Current Frame's Edges (b) Edges' Background Esti-

mation
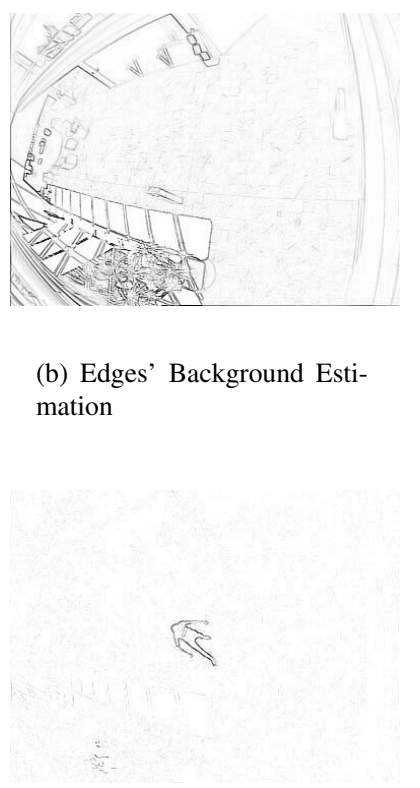

(d) Subtraction of Background from the Current Edges

\section{零 展- \\ (e) Resulted Image}

Figure 2. The steps of the algorithm.

Figure 2 shows some steps of the proposed algorithm. In 2(a) it is shown the original frame $f_{i}$; in 2(b), the background's model $b c k_{i}^{s}$; in 2(c), the current frame's edges $\operatorname{grad}_{i}^{\text {ext }}\left(f_{i}\right)$; in $2(\mathrm{~d})$, the subtraction's result $f e_{i}$ and, in 2(e), the final result $q t_{i}$.

\section{Experimental Results}

The implementation has been done in Matlab with the SDC Morphology Toolbox for Matlab [5]. The algorithm was tested using the videos of the CAVIAR project [8]. These video sequences are divided in two sets. The first set has been acquired with a wide angle camera in the entrance lobby of the INRIA Labs (France) in July, 2003. The second set has been acquired in a mall in Portugal using a wide angle camera. All the video sequences have a resolution of 384 x 288 pixels at 25 frames per second. The image sequences have from 500 to 1400 frames in length and are in MPEG format.

The first set shows actors walking alone, walking together in a group, meeting another person, a group of people being split, simulating a fight, leaving a package in the scene and falling in the ground. There are some challenging features in the videos like a person behind plants in difficult position to be seen and illuminated and dark regions. These videos have low-quality with lots of noise.

The videos in the second set show real customers in a mall. There are frontal and lateral views of a corridor and the sequences show the same time frame in both views. In these videos, people are walking in the mall, entering and exiting shops, meeting another group, walking and stopping suddenly, passing out and changing direction.

No information other than the sequences has been used in the proposed algorithm. There are, of course, some basic assumptions like the sequence being acquired by a single and static camera implying that the background is static, no zoom effects is being applied and etc. Our algorithm returns almost complete edges of the moving objects in all videos. The noise was almost completely eliminated in all videos, even in the low-quality videos. Our algorithm is completely operational since the beginning of the image sequence and it has a 3-frames delay to detect a new object in the scene due to the temporal coherence used in the algorithm.

To initially compare our results to the literature, we have implemented four different algorithms:

a Two frames differentiation: this is an algorithm based on the temporal differentiation algorithm approach. It computes the symmetrical difference among the corresponding pixel values of two consecutive frames and then applies a threshold. Pixels (values) greater than the threshold belongs to the foreground.

b Two frames differentiation plus a morphological filter: this is the same of the algorithm a plus a connected morphological filter that eliminates components with area less than a specified threshold.

c Background subtraction: This is an implementation of the background model subtraction approach. Our implementation can estimate the model using mean, median, mode or adaptive mean, as described in [4]. The foreground segmentation is the result of a threshold of the symmetrical difference between the current frame and the estimated model.

d Hybrid algorithm: it uses a hybrid approach [4]. The algorithm implemented uses a three-frame differentiation, an estimation of the moving areas and, finally a subtraction of a background model, as proposed in [4]. 


\section{"}

(a) Background Subtraction's Results

(b) Our Results
The three-frame differentiation implements the formula, $T h\left(\left|f_{i}-f_{i-1}\right|, h_{1}\right) \wedge T h\left(\left|f_{i}-f_{i-2}\right|, h_{1}\right)$, where $h_{1}$ is a threshold empirically specified. The estimation of the moving areas is done by computing, for each connected component of the three-frame differentiation result, the smallest rectangle that contains it. Finally, the symmetrical difference between this area and the background model is computed. The background model is similar to the one used before but now it is adaptive and uses a learning parameter $\alpha$ that is defined heuristically. Formally, it implements the formula $b c k_{i+1}=\alpha * f_{i}+(1-\alpha) * b c k_{i}$, where $\alpha$ is usually $0.05 \leq \alpha \leq 0.15$. Connected components inside the area of interest that have pixel values similar to the model are eliminated. The remaining pixels are considered foreground.

The overall results of algorithm a show poor shape lateral regions of the moving objects (due to the displacement between consecutive frames). The first set of sequences are very low-quality and lots of noise appear in the results. This algorithm has the best time performance, mainly due to its simplicity. The difference between algorithm $a$ and $b$ is the noise reduction due to the connected filter.

The overall results of algorithm c have good shape recovery. However, the approach is very sensible to the statistics used in the estimation of the background. Median or mode have shown to be more robust in the tested sequences. The results are also very sensible to shadows cast that is a real problem in the sequences taken in the mall.

The overall results of algorithm $\mathrm{d}$, the hybrid algorithm, have a good shape recovery with less noise. However, cast shadows is still a problem in the results. The results in the first set of videos are worse due to bad quality of them. One difficult of this algorithm is to set a good value to the learning rate $\alpha$. As some tested sequences shows many different patterns of movement, a single learning rate does not work well in some parts of the sequences.

The overall results of the proposed algorithm show al- most all edges of the foreground objects. The noise is low, even in the low-quality videos. As the algorithm uses the edges of objects in the frames and not the pixels' intensity, it also shows a robustness to lighting changes. In some situations, like in indoor sequences, this robustness implied in a desirable result: cast shadows do not appeared in the results. Figure 3 illustrates this situation showing cast shadows in the results of background's subtractions 3(a) compared to our results 3 (b) in an indoor mall sequence. If $V_{\min }$ and $H_{\text {min }}$ are too high, parts of the real borders are eliminated . If they are too low, noise is not adequately eliminated. The parameters set used in the algorithm have been experimentally chosen and they are robust in the sense that the same parameters are used for all sequences in both sets. The results are very similar in quality for both video sets. The algorithm is completely operational since the beginning of the image sequence and it has a 3-frames delay to detect a new object in the scene due to the temporal coherence used in the algorithm. When the mean is used for the background estimation, the time performance of the algorithm is about $40 \%$ better compared to median's but this is compensated by a result with little noise. If this is not a problem for the application, mean background estimation can be a plus.

Another interesting part of the proposed algorithm is the absence of false positives when objects that stay still for long periods of time start moving again. Usually, all background subtraction methods fail with this situation because the still object becomes part of the background and, when it starts moving again, an artifact appears in the result (because it is the result of the symmetrical difference between the new images and the old background). The artifact will eventually disappear as the background will be updated. The proposed algorithm has a different behavior, as it uses a simple subtraction among the edges of the current frame and the edges of the background model. The results will not have false positives because of motionless objects that start moving. Figure 4 shows the situation described above comparing a background (estimated by median) subtraction algorithm to the proposed algorithm. Figure 4(a) shows the frame 101 of a tested sequence, Fig. 4(b) shows the background estimated by median of the same frame, Fig. 4(c) shows the result of the background subtraction algorithm, a false positive is in the result in the same place that the person remained motionless for a long time, Fig. 4(d) shows the background used by our algorithm, Fig. 4(e) shows the edges of the same frame and Fig. 4(f) shows our result without the false positive.

Figure 5 shows our results compared to another tested algorithms. Figure 5(a) shows the original frame, Fig. 5(b), the results of the algorithm b, Fig. 5(c) shows results of the background's subtraction, Fig. 5(d) shows the hybrid's results and, finally, in Fig. 5(e) our results are shown. 


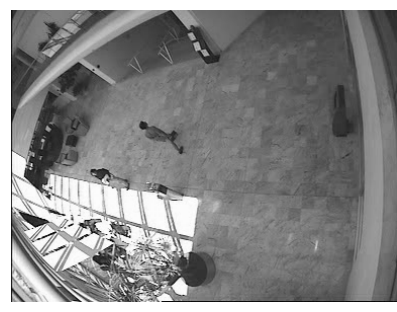

(a) Frame 101 original

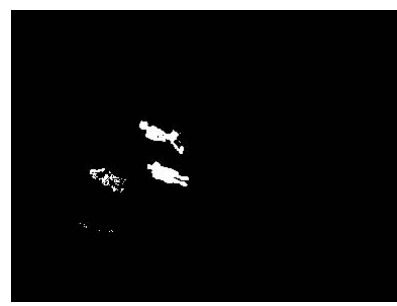

(c) Targets in background subtraction

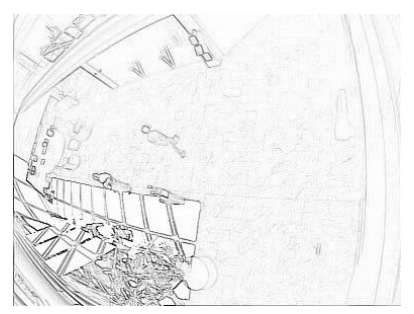

(e) Edges of frame 101

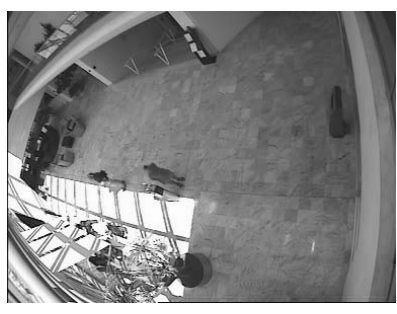

(b) Background estimated by median

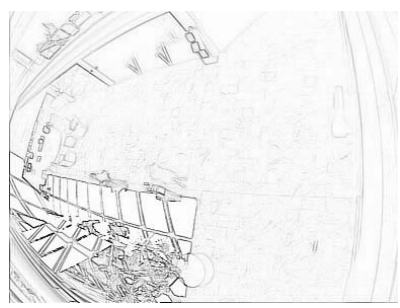

(d) Our background

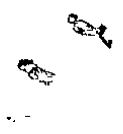

(f) Our result
Figure 4. Showing the absence of false positives in our result.

All results reported here are available at http://www.vision.ime.usp.br/nonlinear/demos/segmm/.

\section{Conclusion}

We have proposed a new algorithm based on Mathematical Morphology to segment moving objects in an image sequence. The algorithm is simple to implement and the overall performance results are promising. In terms of robustness, results are similar in both video sets tested [10], independently of the video's quality. The algorithm is also robust to lighting changes as it uses the edges of objects in the scene and not the pixels' intensity. In some situations, the results have almost no cast shadows. When compared

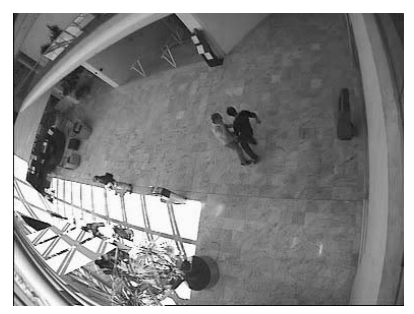

(a) Original Frame

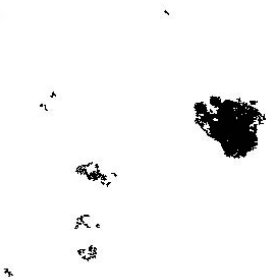

(c) Background's Subtraction

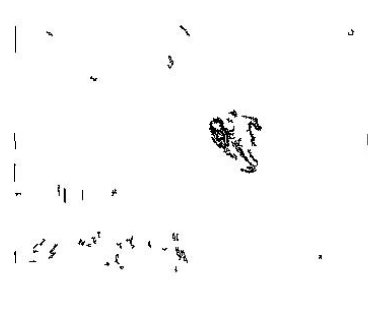

(b) 2-Frames Differentiation

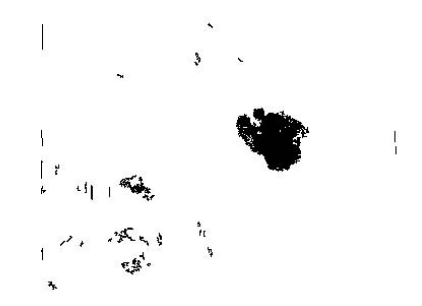

(d) Hybrid

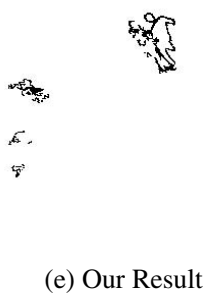

Figure 5. Comparing algorithms.

to other algorithms, our algorithm has shown a good noise reduction.

Another advantage of our algorithm is that there is no distinction among training and running sets as some algorithms reported in the literature. The algorithm is operational since the firsts frames of the image sequence, it is not necessary to have an initial period to train the algorithm. This makes the algorithm more robust and it can also be used in uncontrolled scenes where there is no way to have a sequence without target objects like in a train station or in a full-crowded corner (results shown in our website).

When compared to the background's subtraction algorithms, our algorithm shows an interesting result that is the absence of false foreground, a situation that can happen when a person or an object is motionless (becoming part of the background) and suddenly starts moving.

Using just the edges of the frames, the obtained results 
are encouraging. The next steps will be to implement a complete shape recovery of the foreground's objects. After that, we are planning to test our results in video sets with challenging situations as exemplified in [23] and to measure the error found in that situations.

\section{Acknowledgment}

The authors would like to thank for the public downloadable data that comes from the EC Funded CAVIAR project/IST 2001 37540, found at URL: http://homepages.inf.ed.ac.uk/rbf/CAVIAR/. Arnaldo Câmara Lara is supported by $\mathrm{CNPq}$, the Brazilian National Research Council, via fellowship, process number 131407/2005-8. Roberto Hirata Jr. is partially supported by CNPq.

\section{References}

[1] J. Amat, A. Casals, and M. Frigola. Stereoscopic system for human body tracking in natural scenes. In Proc. IEEE Int. Work. on Modelling People, 1999.

[2] G. J. F. Banon and J. Barrera. Decomposition of Mappings between Complete Lattices by Mathematical Morphology, Part I. General Lattices. Signal Processing, 30:299-327, 1993.

[3] J. Barrera, G. J. F. Banon, R. A. Lotufo, and R. Hirata Jr. Mmach: A mathematical morphology toolbox for khoros system. Journal of Eletronic Imaging, 7(1), January 1998.

[4] R. T. Collins, A. J. Lipton, T. Kanade, H. Fujiyoshi, D. Duggins, Y. Tsin, D. Tolliver, N. Enomoto, O. Hasegawa, P. Burt, and L. Wixson. A system for video surveillance and monitoring. Technical report, Robotics Inst., Carnegie-Mellon Univ., Pittsburgh, PA, 2000. Tech. Rep. CMU-RI-TR-0012.

[5] E. R. Dougherty and R. A. Lotufo. Hands-On Morphological Image Processing. SPIE Press, Bellingham, WA, USA, 2003.

[6] J. M. Ferryman, S. J. Maybank, and A. D. Worral. Visual surveillance for moving vehicles. Int. Journal of Computer Vision, 37(2), June 2000.

[7] C. Finch. Special Effects: Creating Movie Magic. Abbeville Press, 1984.

[8] R. B. Fisher. Pets04 surveillance ground truth data set. In Proc. Sith IEEE Int. Work. on Performance Evaluation of Tracking and Surveillance, May 2004.

[9] R. C. Gonzalez and R. E. Woods. Digital Image Processing. Addison-Wesley Publishing Company, second edition, 2002.

[10] D. Hall, P. Ribeiro, E. Andrade, P. Moreno, S. Pesnel, T. List, R. Emonet, R. B. Fisher, J. S. Victor, and J. L. Crowley. Comparison of target detection algorithms using adaptative background models. In Proc. 2nd Joint IEEE Int. Workshop on Visual Surveillance and Performance Evaluation of Tracking and Surveillance, October 2005.
[11] I. Haritaoglu, D. Harwood, and L. S. Davis. W4: Who? when? where? what? a real time system for detecting and tracking people. In 3rd. Int. Conf. on Face \& Gesture Recognition, 1998.

[12] W. Hu, T. Tan, L. Wang, and S. Maybank. A survey on visual surveillance of object motion and behaviors. IEEE Trans. on Systems, Man, and Cybernetics-Part C: Applic. and Reviews, 34(3), August 2000.

[13] T. Kanade, R. T. Collins, A. J. Lipton, P. Burt, and L. Wixson. Adv. in cooperative multi-sensor video surveillance. In Proc. DARPA Image Understanding Workshop, 1998.

[14] Y. Lu, W. Ga, and F. Wu. Automatic video segmentation using a novel background model. In IEEE Int. Symposium on Circuits and System, volume 3, 2002.

[15] G. Matheron. Random Sets and Integral Geometry. John Wiley, New York, 1975.

[16] Y. Matsushita, K. Nishino, K. Ikeuchi, and M. Sakauchi. Illumination normalization with time-dependent intrinsic images for video surveillance. IEEE Trans. on Pattern Analysis and Machine Intelligence, 26(10), October 2004.

[17] T. B. Moeslund and E. Granum. A survey of computer vision-based human motion capture. Computer Vision and Image Understanding, 81(3), 2001.

[18] J. C. Nascimento, M. A. T. Figueiredo, and J. S. Marques. Motion segmentation for activity surveillance. In 1st Work. on Systems, Decision and Control Robotic Monitoring and Surveillance, Lisbon, June 2005.

[19] R. J. Radke, S. Andra, O. Al-Kofabi, and B. Roysam. Image change detection algorithms: A systematic survey. IEEE Trans. on Image Processing, 14(3), March 2005.

[20] J. Serra. Image Analysis and Mathematical Morphology. Academic Press, London, 1982.

[21] S. M. Smith and J. M. Brady. Asset-2: Real time motion segmentation and shape tracking. IEEE Trans. on Pattern Analysis and Machine Inteligence, 17(8), August 1995.

[22] E. Stringa. Morphological change detection algorithms for surveillance applications. In Proc. British Machine Vision Conf., 2000.

[23] K. Toyama, J. Krumm, B. Brumitt, and B. Meyers. Wallflower: Principles and practice of background maintenance. In 7th Int. Conf. on Computer Vision (ICCV99), volume $1,1999$.

[24] L. Wang, W. Hu, and T. Tan. Recent developments in human motion analysis. Pattern Recognition, 36(3), 2003.

[25] C. R. Wren, A. Azerbayejani, T. Darrell, and A. P. Pentland. Pfinder: Real-time tracking of the human body. IEEE Trans. on Pattern Analysis on Machine Intelligence, 19(7), July 1997. 